\title{
Robust output feedback consensus for networked negative-imaginary systems
}

DOI:

10.1109/TAC.2015.2395472

\section{Document Version}

Accepted author manuscript

Link to publication record in Manchester Research Explorer

\section{Citation for published version (APA):}

Wang, J., Lanzon, A., \& Petersen, I. R. (2015). Robust output feedback consensus for networked negativeimaginary systems. I E E E Transactions on Automatic Control, 60(9), 2547-2552.

https://doi.org/10.1109/TAC.2015.2395472

\section{Published in:}

I E E E Transactions on Automatic Control

\section{Citing this paper}

Please note that where the full-text provided on Manchester Research Explorer is the Author Accepted Manuscript or Proof version this may differ from the final Published version. If citing, it is advised that you check and use the publisher's definitive version.

\section{General rights}

Copyright and moral rights for the publications made accessible in the Research Explorer are retained by the authors and/or other copyright owners and it is a condition of accessing publications that users recognise and abide by the legal requirements associated with these rights.

\section{Takedown policy}

If you believe that this document breaches copyright please refer to the University of Manchester's Takedown Procedures [http://man.ac.uk/04Y6Bo] or contact uml.scholarlycommunications@manchester.ac.uk providing relevant details, so we can investigate your claim.

\section{OPEN ACCESS}




\section{Robust Output Feedback Consensus for Networked Negative-Imaginary Systems}

\author{
Jianan Wang, Member, IEEE, Alexander Lanzon, Senior \\ Member, IEEE, and Ian R. Petersen, Fellow, IEEE
}

\begin{abstract}
A robust output feedback consensus problem for networked homogeneous Negative-Imaginary (NI) systems is investigated in this technical note. By virtue of NI systems theory, a set of reasonable yet elegant conditions are derived for output consensus under $\mathfrak{L}_{2}$ externa disturbances as well as NI model uncertainty. As a byproduct, this technical note also reaffirms a previous result in [1] which shows the robustness of networked systems is always worse than that of single agent system. Furthermore, the eventual convergence sets are also characterised for several special NI systems that are commonly studied in the literature. It is shown how the results in this work embed and generalise earlier results for these classes of systems. We show that the natural convergence set boils down to the centroid of the initial pattern when the initia conditions of the controllers are zero. Numerical examples are given to showcase the main results.
\end{abstract}

Index Terms-Consensus, Cooperative Control, Negative-Imaginary Systems, Robust Control.

\section{INTRODUCTION}

NI systems are, broadly speaking, systems with a negative imaginary frequency response. This class of systems has received extensive attention in recent years [2], [3] since it was introduced in [4] and found its most successful application in the area of nanopositioning control [5] where co-located force actuation and position measurement are typical [6]. NI systems theory has also been widely applied to the control of flexible structures with highly-resonan dynamics, which is typically a challenging task to tackle via classical methods. Robust stability analysis of interconnected systems with mixed NI and small-gain properties has also been studied in [7].

The area of cooperative control has been very active over the past decade and it was immediately evident that distributed control and communication networks play an important role in stability analysis. The output feedback consensus problem, or more precisely, the output synchronization problem was first studied in [8], and a solution for weakly minimum phase nonlinear systems with relative degree one was presented. Later, [9] extended the result to heterogeneous cases even with uncertainties. The output feedback consensus problem that we consider is to have all the outputs naturally converge to a common trajectory (not necessarily constant) which is entirely determined by the subsystems themselves as well as the graph properties. Although similar approaches were presented in [1], [10] and [11] using a statespace representation, this work can be distinguished from these works via the following aspects: (a) a much simpler D.C. gain condition for robust output feedback consensus is given, while the aforementioned works mainly build on the existence of a matrix or matrices such that the error dynamics are stable, which is usually hard to find; (b) [10] and [11] do not study a robust control law, whereas this article does;

J. Wang was previously with Control Systems Centre, School of Electrical and Electronic Engineering, University of Manchester, Manchester M13 9PL, UK, and is now with Key Laboratory of Dynamics and Control of Flight Vehicle within Ministry of Education, School of Aerospace Engineering, Beijing Institute of Technology, Beijing 100081, China (e-mail: wangjianan@ieee.org).

A. Lanzon are with Control Systems Centre, School of Electrical and Electronic Engineering, University of Manchester, Manchester M13 9PL, UK (e-mail: a.lanzon@ieee.org).

Ian R. Petersen is with School of Information Technology and Electrical Engineering, University of New South Wales, Australian Defence Force Academy, Canberra, A.C.T. 2600, Australia (e-mail: i.r.petersen@gmail.com). Manuscript received on January 16, 2015. (c) this work also captures the result of [1] regarding the robustness of the multi-agent systems is never better than that of single agent systems. Recently, a robust consensus problem for heterogeneous multi-agent systems was discussed in [12]. However, the agents considered are constrained to second-order systems, which is just an example of NI systems and the consensus algorithm is based on full state information which is infeasible in most cases, whereas here we handle output feedback. Another work [13] addressed an output consensus problem of heterogeneous uncertain linear multi-agent systems. However, this work requires the following assumptions: (a) [13] makes a minimum phase assumption on all plants which allows the use of high gain control whereas the NI systems in this work are not necessarily minimum phase; (b) [13] only studies a class of unmodelled dynamics but does not explicitly tackle $\mathfrak{L}_{2}$ external disturbances whereas this work studies both; (c) again, [13] deals with an output synchronization problem to a limited class of trajectories, such as constant, sinusoidal and diverging signals which are polynomial functions of time due to technical reasons whereas this work studies a consensus problem naturally converging to an unspecified trajectory.

This technical note is motivated by applications in which the system goal cannot be accomplished by a single NI system due to limitations in its capability, such as coverage or precision. This in turn requires the coordination of multiple NI systems, which in this work involves output feedback consensus under external disturbances and model uncertainty. In this technical note, a homogeneous network of NI systems and a fixed communication topology are assumed. The $i$ th NI system is described in the $s$-domain:

$$
\boldsymbol{y}_{i}=P(s) \boldsymbol{u}_{i}, i=1, \cdots, n
$$

where $P(s)$ is the transfer function (generally MIMO), $\boldsymbol{y}_{i} \in \mathbb{R}^{m \times 1}$ and $\boldsymbol{u}_{i} \in \mathbb{R}^{m \times 1}$ are the output and input of the system with the dimension $m \geq 1, n>1$ is the number of agents. Then, an elegant problem formulation, using the Laplacian matrix and Kronecker product, is adopted such that the output feedback consensus problem is cast into a robust stability problem, which can be solved via NI systems theory as detailed in [4], [14] and [15]. The contributions of this technical note can be summarized as follows: (a) it provides a novel viewpoint where consensus problems can be studied as internal stability problems, (b) it only exploits output feedback information as opposed to the full state feedback which is common in the literature, (c) it gives a class of consensus protocols that can be tuned for performance and/or robustness, (d) it provides a robustness guarantee via NI systems theory, and (e) it characterises the convergence sets. Notation: $\mathbb{R}^{m \times n}$ and $\mathbb{C}^{m \times n}$ denote the sets of $m \times n$ real and complex matrices respectively. $I_{n}$ is the $n \times n$ identity matrix and $\mathbf{1}_{n}$ is the $n \times 1$ vector with all elements being 1 . Given $M \in \mathbb{R}^{n \times n}$, $M>(<) 0$ means $M$ is positive (negative) definite and $M \geq(\leq) 0$ means $M$ is positive (negative) semi-definite. $\bar{\lambda}(M)$ denotes the largest eigenvalue of $M$ when $M$ has only real eigenvalues and $\bar{\sigma}(M), \underline{\sigma}(M)$ represent the maximum and minimum singular values of $M$ respectively. $\mathbb{N}(M)$ denotes the null space of $M . M^{T}$ and $M^{*}$ are the transpose and the complex conjugate transpose of $M$. In addition, given $s \in \mathbb{C}, \operatorname{Re}[s]$ is the real part of $s$. Given $a_{1}, a_{2} \in \mathbb{C}$, $\operatorname{diag}\left(a_{1}, a_{2}\right)=\left[\begin{array}{cc}a_{1} & 0 \\ 0 & a_{2}\end{array}\right]$. Finally, given $\boldsymbol{a} \in \mathbb{R}^{n \times 1}$, ave $(\boldsymbol{a})$ is the average operation of all elements of $\boldsymbol{a}$. OLHP is short for open left half plane and MIMO is short for multi-input and multi-output.

Preliminaries of graph theory: A graph can be mathematically expressed by $\mathcal{G}=(\mathcal{V}, \mathcal{E})$ where $\mathcal{V}=\left\{v_{1}, v_{2}, \ldots, v_{n}\right\}$ is a nonempty finite set of $n$ nodes and an edge set $\mathcal{E} \subseteq \mathcal{V} \times \mathcal{V}$ is used to model the communications links among nodes. The adjacency matrix $\mathcal{A}=\left[a_{i j}\right] \in \mathbb{R}^{n \times n}$, where $a_{i i}=0$ and $\forall i, j$ with $i \neq j$, 
$a_{i j}=1$ if $\left(v_{i}, v_{j}\right) \in \mathcal{E}$ and 0 otherwise. The in-degree of node $i$ is defined as $d_{i}=\sum_{j} a_{i j}$ and $\mathcal{D}=\operatorname{diag}\left\{d_{1}, d_{2}, \cdots, d_{n}\right\} \in \mathbb{R}^{n \times n}$ is the in-degree matrix. The Laplacian matrix of graph $\mathcal{G}$ is given by $\mathcal{L}_{n}=\mathcal{D}-\mathcal{A}$. A sequence of successive edges of $\mathcal{E}$ in the form of $\left\{\left(v_{i}, v_{k}\right),\left(v_{k}, v_{l}\right), \ldots,\left(v_{m}, v_{j}\right)\right\}$ is defined as a path from node $i$ to node $j$. An undirected graph is said to be connected if there is a path from node $i$ to node $j$ for all the distinct nodes $v_{i}, v_{j} \in \mathcal{V}$. It is well-known that $\mathcal{L}_{n}$ has the following properties when the graph is undirected and connected:

$$
\mathcal{L}_{n} \geq 0, \mathbb{N}\left(\mathcal{L}_{n}\right)=\operatorname{span}\left\{\mathbf{1}_{n}\right\} .
$$

\section{Robust Output Feedback Consensus Protocol}

In this section, a class of output feedback consensus protocols for networked NI systems under external disturbances and NI model uncertainty is considered. To this end, let us first recall the definitions of NI and SNI (short for Strictly Negative-Imaginary) systems:

Definition 1: ([15]) A square, real, rational, proper transfer function $P(s)$ is NI if the following conditions are satisfied:

1) $P(s)$ has no pole in $\operatorname{Re}[s]>0$;

2) $\forall \omega>0$ such that $j \omega$ is not a pole of $P(s), j(P(j \omega)-$ $\left.P(j \omega)^{*}\right) \geq 0$

3) If $s=j \omega_{0}$ with $\omega_{0}>0$ is a pole of $P(s)$, then it is a simple pole and the residue matrix $K=\lim _{s \rightarrow j \omega_{0}}\left(s-j \omega_{0}\right) j P(s)$ is Hermitian and positive semi-definite;

4) If $s=0$ is a pole of $P(s)$, then $\lim _{s \rightarrow 0} s^{k} P(s)=0, \forall k \geq 3$ and $P_{2}=\lim _{s \rightarrow 0} s^{2} P(s)$ is Hermitian and positive semi-definite.

It can be observed that Definition 1 captures the definitions of NI systems in [4] and [14]. Examples of NI systems can be found in [6] and include single-integrator systems, double-integrator systems, undamped and damped flexible structures, to name a few typically considered in the consensus literature.

Definition 2: ([4]) A square, real, rational, proper transfer function $P_{s}(s)$ is SNI if the following conditions are satisfied:

1) $P_{s}(s)$ has no pole in $\operatorname{Re}[s] \geq 0$;

2) $\forall \omega>0, j\left(P_{s}(j \omega)-P_{s}(j \omega)^{*}\right)>0$.

Homogeneous NI agents are defined in the $s$-domain in the form of (1). Since $P(s)$ is in general a MIMO plant, the Laplacian matrix describing the network interconnection is modified via a Kronecker product to $\mathcal{L}_{n} \otimes I_{m}$ and the total networked plant under consideration is depicted in Fig. 1 with

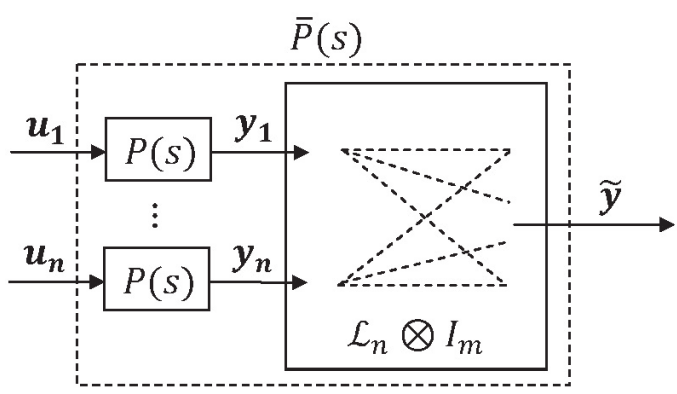

Fig. 1. Networked NI systems

$$
\tilde{\boldsymbol{y}}=\bar{P}(s) \boldsymbol{u}=\left(\mathcal{L}_{n} \otimes I_{m}\right)\left(I_{n} \otimes P(s)\right) \boldsymbol{u}=\left(\mathcal{L}_{n} \otimes P(s)\right) \boldsymbol{u},
$$

where $\bar{P}(s)$ is the augmented plant, $\boldsymbol{y}=\left[\boldsymbol{y}_{1}^{T}, \cdots, \boldsymbol{y}_{n}^{T}\right]^{T} \in \mathbb{R}^{n m \times 1}$ and $\boldsymbol{u}=\left[\boldsymbol{u}_{1}^{T}, \cdots, \boldsymbol{u}_{n}^{T}\right]^{T} \in \mathbb{R}^{n m \times 1}$. In general, robust output feedback consensus is defined as follows:

Definition 3: A distributed output feedback control law achieves robust output feedback consensus for a network of systems when
1) output consensus is achieved, i.e., $\boldsymbol{y}_{i} \rightarrow \boldsymbol{y}_{s s}, \forall i \in\{1, \cdots, n\}$ for a family of plant dynamics with no external disturbance, where $\boldsymbol{y}_{s s}$ is the final convergence trajectory.

2) $\boldsymbol{y}_{s s}$ is perturbed by additive $\mathfrak{L}_{2}[0, \infty]$ signals when $\mathfrak{L}_{2}[0, \infty]$ disturbances are present on both input and output.

It can be seen that the output $\boldsymbol{y}$ reaches consensus when $\tilde{\boldsymbol{y}} \rightarrow \mathbf{0}$ via the properties of the Laplacian given in (2). This formulation actually converts the output consensus problem to an internal stability problem which is easier to tackle and investigate the robustness property via standard control theoretic methods. We now impose the following standing assumption:

Assumption 1: $\mathcal{G}$ is undirected and connected.

The following preliminary lemmas are needed:

Lemma 1: ([16]) Let $\lambda_{j}$ and $\gamma_{k}, j=1, \cdots, n, k=1, \cdots, m$, be eigenvalues of matrices $\Lambda_{n \times n}$ and $\Gamma_{m \times m}$ respectively, the eigenvalues of $\Lambda \otimes \Gamma$ are $\lambda_{j} \gamma_{k}$.

Note that Lemma 1 also applies to the singular values [16].

Lemma 2: Given $\Lambda \in \mathbb{R}^{n \times n}$ and $\Gamma \in \mathbb{R}^{m \times m}$, then

$$
\begin{aligned}
\mathbb{N}(\Lambda \otimes \Gamma)= & \left\{\boldsymbol{a} \otimes \boldsymbol{b}: \boldsymbol{b} \in \mathbb{R}^{m \times 1}, \boldsymbol{a} \in \mathbb{N}(\Lambda)\right\} \\
& \cup\left\{\boldsymbol{c} \otimes \boldsymbol{d}: \boldsymbol{c} \in \mathbb{R}^{n \times 1}, \boldsymbol{d} \in \mathbb{N}(\Gamma)\right\} .
\end{aligned}
$$

Proof: the proof simply follows from the definition of null space and the properties of Kronecker product.

The following lemma states that the augmented networked plant $\bar{P}(s)=\mathcal{L}_{n} \otimes P(s)$ is NI if and only if every single system $P(s)$ is NI.

Lemma 3: $\bar{P}(s)$ is NI if and only if $P(s)$ is NI.

Proof: First note that $\mathcal{L}_{n} \geq 0$ due to Assumption 1 in (3). Then, the sufficiency and necessity are straightforward by applying Lemma 1 to Definition 1.

Since Lemma 3 requires positive semi-definiteness of $\mathcal{L}_{n}$, this work cannot be applied to directed graphs. The output $\tilde{\boldsymbol{y}} \rightarrow \mathbf{0}$ if internal stability is achieved for $\bar{P}(s)$ with some controller. From [4], [14] and [15], the following internal stability results are summarized:

Lemma 4: Given an NI transfer function $P(s)$ and an SNI transfer function $P_{s}(s)$ with $P_{2}=\lim _{s \rightarrow 0} s^{2} P(s), P_{1}=\lim _{s \rightarrow 0} s\left(P(s)-\frac{P_{2}}{s^{2}}\right)$ and $P_{0}=\lim \left(P(s)-\frac{P_{2}}{s^{2}}-\frac{s \rightarrow 0}{s}\right)$, the positive feedback interconnection $\left[P(s), \vec{P}_{s}(s)\right]$ is internally stable if and only if any of the following conditions is satisfied:

1) $\bar{\lambda}\left(P(0) P_{s}(0)\right)<1$ when $P(s)$ has no pole(s) at the origin, $P(\infty) P_{s}(\infty)=0$ and $P_{s}(\infty) \geq 0$;

2) $J^{T} P_{s}(0) J<0$ when $P(s)$ has pole(s) at the origin and is strictly proper, $P_{2} \neq 0, P_{1}=0, \mathbb{N}\left(P_{2}\right) \subseteq \mathbb{N}\left(P_{0}^{T}\right)$, where $P_{2}=$ $J J^{T}$ with $J$ having full column rank;

3) $F_{1}^{T} P_{s}(0) F_{1}<0$ when $P(s)$ has pole(s) at the origin and is strictly proper, $P_{2}=0, P_{1} \neq 0, \mathbb{N}\left(P_{1}^{T}\right) \subseteq \mathbb{N}\left(P_{0}^{T}\right)$, where $P_{1}=F_{1} V_{1}^{T}$ with $F_{1}$ and $V_{1}$ having full column rank and $V_{1}^{T} V_{1}=I$.

Note that the above result is actually a robust stability result because an NI plant $P(s)$ can be perturbed by any unmodelled dynamics $\Delta(s)$ such that the perturbed plant $P_{\Delta}(s)$ which then replaces the nominal plant $P(s)$ in Lemma 4 retains the NI system property and still fulfills any one of the conditions in Lemma 4. Similarly, $P_{s}(s)$ can be perturbed to any SNI controller subject to 1), 2), 3). Henceforth, we do not distinguish between $P(s)$ and $P_{\Delta}(s)$ for simplicity of notation, though it is stressed that $P(s)$ could be the resulting perturbed dynamics of some simpler nominal plant. There is clearly a huge class of permissible dynamic perturbations to the nominal dynamics as conditions 1), 2) and 3) impose a restriction on $P(s)$ only at the frequency $\omega=0$ or on the associated residues of $P(s)$ at $\omega=0$ and the NI class has no gain or order restriction [4]. A few examples of permissible perturbations are 
additive perturbations where the uncertainty is also NI [4], feedback perturbations where both systems in the feedback interconnection are NI [6] and more general perturbations based on Redheffer Starproducts and Linear Fractional Transformations [6]. For example, $\frac{1}{s+5}$ and $\frac{\left(2 s^{2}+s+1\right)}{\left(s^{2}+2 s+5\right)(s+1)(2 s+1)}$ are both NI with the same D.C. gain. Now, we are ready to state the first main result of this work:

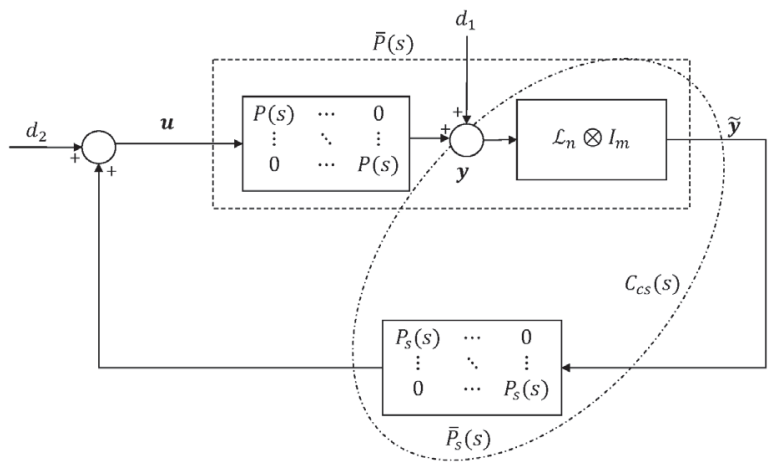

Fig. 2. Closed-loop system with SNI controllers

Theorem 1: Given a graph $\mathcal{G}$ which satisfies Assumption 1 and models the communication links for networked homogeneous NI systems, and given any SNI control law $P_{s}(s)$, robust output feedback consensus is achieved via the protocol

$$
\boldsymbol{U}_{c s}=\bar{P}_{s}(s) \tilde{\boldsymbol{y}}=C_{c s}(s) \boldsymbol{y}=\left(\mathcal{L}_{n} \otimes P_{s}(s)\right) \boldsymbol{y}
$$

shown in Fig. 2, or in a distributed manner for each agent $i$,

$$
\boldsymbol{u}_{i}=P_{s}(s) \sum_{j=1}^{n} a_{i j}\left(\boldsymbol{y}_{i}-\boldsymbol{y}_{j}\right)
$$

under any external disturbances $\boldsymbol{d}_{1}, \boldsymbol{d}_{2} \in \mathfrak{L}_{2}[0, \infty)$ and any model uncertainty which retains the NI system property of the perturbed plant $P(s)$ if and only if $P(s)$ and $P_{s}(s)$ satisfy 1$\left.), 2\right)$ or 3 ) in Lemma 4 except that

$$
\bar{\lambda}\left(P(0) P_{s}(0)\right)<\frac{1}{\bar{\lambda}\left(\mathcal{L}_{n}\right)}
$$

replaces $\bar{\lambda}\left(P(0) P_{s}(0)\right)<1$ in case 1$)$.

Proof: Before presenting the consensus result, let us first prove the internal stability of $\left[\bar{P}(s), \bar{P}_{s}(s)\right]$ using Lemma 4. From Fig. 2, we have $\bar{P}(s)=\mathcal{L}_{n} \otimes P(s)$ which has been shown to be NI in Lemma 3 and it is straightforward to see $\bar{P}_{s}(s)=I_{n} \otimes P_{s}(s)$ is SNI since $P_{s}(s)$ is SNI.

$(\Leftarrow)$ Sufficiency: From Lemma 4, we can conclude that $\left[\bar{P}(s), \bar{P}_{s}(s)\right]$ is internally stable since

1) when $P(s)$ has no pole(s) at the origin, $\bar{P}(s)$ has no pole(s) at the origin as well. Also, $\bar{P}(\infty) \bar{P}_{s}(\infty)=\left(\mathcal{L}_{n} \otimes\right.$ $P(\infty))\left(I_{n} \otimes P_{s}(\infty)\right)=\mathcal{L}_{n} \otimes\left(P(\infty) P_{s}(\infty)\right)=0$ and $\bar{P}_{s}(\infty)=\mathcal{L}_{n} \otimes P_{s}(\infty) \geq 0$ due to Lemma 1 as well as the pair of $\left[P(s), P_{s}(s)\right]$ satisfies condition 1) of Lemma 4 . Finally, $\bar{\lambda}\left(\bar{P}(0) \bar{P}_{s}(0)\right)=\bar{\lambda}\left(\mathcal{L}_{n} \otimes\left(P(0) P_{s}(0)\right)\right)<1$ since $\bar{\lambda}\left(P(0) P_{s}(0)\right)<\frac{1}{\lambda\left(\mathcal{L}_{n}\right)}$ due to Lemma 1 .

2) when $P(s)$ has pole(s) at the origin, $\bar{P}(s)$ has pole(s) at the origin as well. In the case of $P_{2} \neq 0, P_{1}=0$, it is straightforward to see $\mathbb{N}\left(\mathcal{L}_{n} \otimes P_{2}\right)=\mathbb{N}\left(\bar{P}_{2}\right) \subseteq \mathbb{N}\left(\bar{P}_{0}^{T}\right)=\mathbb{N}\left(\mathcal{L}_{n} \otimes P_{0}^{T}\right)$ due to Lemma 2 and $\mathbb{N}\left(P_{2}\right) \subseteq \mathbb{N}\left(P_{0}^{T}\right)$. Furthermore, $\bar{P}_{2}=\mathcal{L}_{n} \otimes P_{2}=$ $\left(J_{\mathcal{L}} J_{\mathcal{L}}^{T}\right) \otimes\left(J J^{T}\right)=\left(J_{\mathcal{L}} \otimes J\right)\left(J_{\mathcal{L}} \otimes J\right)^{T}=\bar{J} \bar{J}^{T}$ since $\mathcal{L}_{n}$ and $P_{2}$ are both Hermitian and positive semi-definite, where $J_{\mathcal{L}}$ has full column rank being $n-1$. With the definition of $\bar{J}=J_{\mathcal{L}} \otimes J$, we have $\bar{J}^{T} \bar{P}_{s}(0) \bar{J}=\left(J_{\mathcal{L}} \otimes J\right)^{T}\left(I_{n} \otimes P_{s}(0)\right)\left(J_{\mathcal{L}} \otimes J\right)=$
$\left(J_{\mathcal{L}}^{T} I_{n} J_{\mathcal{L}}\right) \otimes\left(J^{T} P_{s}(0) J\right)=\left(J_{\mathcal{L}}^{T} J_{\mathcal{L}}\right) \otimes\left(J^{T} P_{s}(0) J\right)<0$ since $J_{\mathcal{L}}^{T} J_{\mathcal{L}}>0$ (with full rank of $n-1$ ) as well as Lemma 1 and condition 2) of Lemma 4

3) The case of $P_{2}=0, P_{1} \neq 0$ follows in the similar manner as case 2) by noting that $\bar{F}_{1}=J_{\mathcal{L}} \otimes F_{1}$.

$(\Rightarrow)$ Necessity is trivial by reversing the above arguments.

The internal stability of $\left[\bar{P}(s), \bar{P}_{s}(s)\right]$ implies output consensus when $\boldsymbol{d}_{1}=\boldsymbol{d}_{2}=\mathbf{0}$, by noting that $\tilde{\boldsymbol{y}} \rightarrow \mathbf{0} \Longleftrightarrow \boldsymbol{y} \rightarrow \mathbf{1}_{n} \otimes \boldsymbol{y}_{s s}$, i.e., $\boldsymbol{y}_{i} \rightarrow \boldsymbol{y}_{s s} \in \mathbb{R}^{m \times 1}$, which is the null space of $\mathcal{L}_{n} \otimes I_{m}$ when $\mathcal{G}$ is undirected and connected.

Robustness to model uncertainty which retains the NI property of $P(s)$ is assured as the result is applicable to any NI plant $P(s)$. Furthermore, the external disturbances $\boldsymbol{d}_{2}, \boldsymbol{d}_{1}$ in Fig. 2 on input $\boldsymbol{u}$ and output $\boldsymbol{y}$ are equivalent to $\boldsymbol{d}_{2},\left(\mathcal{L}_{n} \otimes I_{m}\right) \boldsymbol{d}_{1}$ on input $\boldsymbol{u}$ and output $\tilde{\boldsymbol{y}}$, which is a subset of $\mathfrak{L}_{2}$ disturbances. Hence, the control protocol (4) or (5) will achieve a perturbed $\mathfrak{L}_{2}$ consensus signal on output $\boldsymbol{y}$ (due to superposition principle of linear systems) for all $\mathfrak{L}_{2}$ disturbances $\boldsymbol{d}_{1}, \boldsymbol{d}_{2}$.

Remark 1: It can be seen that the condition in inequality (6) is stricter than that in the inequality of case 1) of Lemma 4 due to the network interconnection. If originally $P_{s}(0)$ was such that $0<$ $\bar{\lambda}\left(P(0) P_{s}(0)\right)<1$, the controller $P_{s}(0)$ needs to be tuned for smaller eigenvalues in order to satisfy inequality (6). On the other hand, if $\bar{\lambda}\left(P(0) P_{s}(0)\right)<0$, there is no need to tune further.

From Fig. 2 and [17], it is convenient to define the input loop transfer matrix, $L_{i}=-\left(I_{n} \otimes P_{s}(s)\right)\left(\mathcal{L}_{n} \otimes P(s)\right)=-\mathcal{L}_{n} \otimes\left(P_{s}(s) P(s)\right)$, and output loop transfer matrix, $L_{o}=-\left(\mathcal{L}_{n} \otimes P(s)\right)\left(I_{n} \otimes P_{s}(s)\right)=$ $-\mathcal{L}_{n} \otimes\left(P(s) P_{s}(s)\right)$, respectively. The input and output sensitivity matrices are defined as $S_{i}=\left(I+L_{i}\right)^{-1}$ and $S_{o}=\left(I+L_{o}\right)^{-1}$. If the closed-loop system is internally stable, the following equations hold:

$$
\begin{aligned}
& \tilde{\boldsymbol{y}}=S_{o}\left(\mathcal{L}_{n} \otimes I_{m}\right) \boldsymbol{d}_{1}+S_{o}\left(\mathcal{L}_{n} \otimes P(s)\right) \boldsymbol{d}_{2} \\
& \boldsymbol{u}=S_{i}\left(\mathcal{L}_{n} \otimes P_{s}(s)\right) \boldsymbol{d}_{1}+S_{i} \boldsymbol{d}_{2} .
\end{aligned}
$$

Good robustness to high frequency unmodelled dynamics is given by the condition in [17]:

$$
\begin{aligned}
& \bar{\sigma}\left(-\mathcal{L}_{n} \otimes P(j \omega) P_{s}(j \omega)\right) \ll 1, \bar{\sigma}\left(-\mathcal{L}_{n} \otimes P_{s}(j \omega) P(j \omega)\right) \ll 1 \\
& \text { and } \bar{\sigma}\left(-I_{m} \otimes P_{s}(j \omega)\right) \ll M \\
\Longleftrightarrow & \bar{\sigma}\left(P(j \omega) P_{s}(j \omega)\right) \ll \frac{1}{\bar{\sigma}\left(\mathcal{L}_{n}\right)}, \bar{\sigma}\left(P_{s}(j \omega) P(j \omega)\right) \ll \frac{1}{\bar{\sigma}\left(\mathcal{L}_{n}\right)} \\
& \text { and } \bar{\sigma}\left(P_{s}(j \omega)\right) \ll \frac{M}{\bar{\sigma}\left(\mathcal{L}_{n}\right)}
\end{aligned}
$$

where $M$ is sufficiently small and $\bar{\sigma}\left(\mathcal{L}_{n}\right)=\bar{\lambda}\left(\mathcal{L}_{n}\right)$ for the undirected and connected graph.

Remark 2: Inequality (8) implies that the robust condition for networked systems is always more stringent than that for a single system by noting that $\bar{\sigma}\left(\mathcal{L}_{n}\right)=\bar{\lambda}\left(\mathcal{L}_{n}\right)>1$ ([18]), which reaffirms the result of [1].

\section{Convergence Set Study}

Section II provides a class of general robust output feedback consensus protocols that guarantees the convergence of the NI systems' outputs $\boldsymbol{y}_{i}$ under external disturbances as well as NI model uncertainty. This section mainly aims at investigating the steady state nominal values of $\boldsymbol{y}_{s s}$ under the proposed output feedback consensus protocol. In order to specify the exact convergence set, the external disturbances and model uncertainty will not be considered in this section.

Given a minimal realization of the $i$ th NI plant $P(s)$,

$$
\left\{\begin{array}{l}
\dot{\boldsymbol{x}}_{i}^{p \times 1}=A^{p \times p} \boldsymbol{x}_{i}^{p \times 1}+B^{p \times m} \boldsymbol{u}_{i}^{m \times 1} \\
\boldsymbol{y}_{i}^{m \times 1}=C^{m \times p} \boldsymbol{x}_{i}^{p \times 1}+D^{m \times m} \boldsymbol{u}_{i}^{m \times 1}
\end{array}, i=1, \cdots, n,\right.
$$


and a minimal realization of the $i$ th SNI controller $P_{s}(s)$,

$$
\left\{\begin{array}{l}
\dot{\overline{\boldsymbol{x}}}_{i}^{q \times 1}=\bar{A}^{q \times q} \overline{\boldsymbol{x}}_{i}^{q \times 1}+\bar{B}^{q \times m} \overline{\boldsymbol{u}}_{i}^{m \times 1} \\
\overline{\boldsymbol{y}}_{i}^{m \times 1}=\bar{C}^{m \times q} \overline{\boldsymbol{x}}_{i}^{q \times 1}+\bar{D}^{m \times m} \overline{\boldsymbol{u}}_{i}^{m \times 1} \quad, i=1, \cdots, n,
\end{array}\right.
$$

where $p$ and $q$ are the dimensions of the states of the NI plant and the SNI controller, respectively. The closed-loop system of Fig. 2 is given as

$$
\begin{aligned}
{\left[\begin{array}{c}
\dot{\overline{\boldsymbol{x}}} \\
\dot{\boldsymbol{x}}
\end{array}\right] } & =\left[\begin{array}{cc}
I_{n} \otimes \bar{A}+\mathcal{L}_{n} \otimes \bar{B} D \bar{C} & \mathcal{L}_{n} \otimes \bar{B} C \\
I_{n} \otimes B \bar{C} & I_{n} \otimes A+\mathcal{L}_{n} \otimes B \bar{D} C
\end{array}\right]\left[\begin{array}{l}
\overline{\boldsymbol{x}} \\
\boldsymbol{x}
\end{array}\right] \\
& \triangleq \Psi\left[\begin{array}{l}
\overline{\boldsymbol{x}} \\
\boldsymbol{x}
\end{array}\right] .
\end{aligned}
$$

The spectrum of $\Psi$ is of importance since it will determine the equilibria. In particular, in this work, the eigenvalues of $\Psi$ on the imaginary axis will determine the steady-state behaviour. To this end, the following lemma is given to characterise the spectrum of $\Psi$

Lemma 5: Let $\lambda_{\mathcal{L}}^{i}$ be the $i$ th eigenvalue of $\mathcal{L}_{n}$ associated with eigenvector $\boldsymbol{v}_{\mathcal{L}}^{i}$. The spectrum of $\Psi$ is given by the union of spectra of the following matrices:

$$
\psi_{i}=\left[\begin{array}{cc}
\bar{A}+\lambda_{\mathcal{L}}^{i} \bar{B} D \bar{C} & \lambda_{\mathcal{L}}^{i} \bar{B} C \\
B \bar{C} & A+\lambda_{\mathcal{L}}^{i} B \bar{D} C
\end{array}\right], i=1, \cdots, n .
$$

Furthermore, let $\left[\boldsymbol{v}_{\mathbf{1}}^{i^{T}} \boldsymbol{v}_{\mathbf{2}}^{i^{T}}\right]^{T}$ be an eigenvector of $\psi_{i}$. Then, the corresponding eigenvector of $\Psi$ is $\left[\begin{array}{l}\boldsymbol{v}_{\mathcal{L}}^{i} \otimes \boldsymbol{v}_{1}^{i} \\ \boldsymbol{v}_{\mathcal{L}}^{i} \otimes \boldsymbol{v}_{2}^{i}\end{array}\right]$.

Proof: Let $\lambda_{\psi_{i}}$ be the eigenvalue of $\psi_{i}$ and

$$
\begin{aligned}
\Psi\left[\begin{array}{c}
\boldsymbol{v}_{\mathcal{L}}^{i} \otimes \boldsymbol{v}_{1}^{i} \\
\boldsymbol{v}_{\mathcal{L}}^{i} \otimes \boldsymbol{v}_{2}^{i}
\end{array}\right] & =\left[\begin{array}{c}
\boldsymbol{v}_{\mathcal{L}}^{i} \otimes\left(\bar{A} \boldsymbol{v}_{1}^{i}+\lambda_{\mathcal{L}}^{i} \bar{B} D \bar{C} \boldsymbol{v}_{1}^{i}+\lambda_{\mathcal{L}}^{i} \bar{B} C \boldsymbol{v}_{2}^{i}\right) \\
\boldsymbol{v}_{\mathcal{L}}^{i} \otimes\left(B \bar{C} \boldsymbol{v}_{1}^{i}+A \boldsymbol{v}_{2}^{i}+\lambda_{\mathcal{L}}^{i} B \bar{D} C \boldsymbol{v}_{2}^{i}\right)
\end{array}\right] \\
& =\left[\begin{array}{c}
\boldsymbol{v}_{\mathcal{L}}^{i} \otimes \lambda_{\psi_{i}} \boldsymbol{v}_{1}^{i} \\
\boldsymbol{v}_{\mathcal{L}}^{i} \otimes \lambda_{\psi_{i}} \boldsymbol{v}_{2}^{i}
\end{array}\right]=\lambda_{\psi_{i}}\left[\begin{array}{c}
\boldsymbol{v}_{\mathcal{L}}^{i} \otimes \boldsymbol{v}_{1}^{i} \\
\boldsymbol{v}_{\mathcal{L}}^{i} \otimes \boldsymbol{v}_{2}^{i}
\end{array}\right]
\end{aligned}
$$

which shows that $\lambda_{\psi_{i}}$ is also an eigenvalue of $\Psi$ with the associated eigenvector being $\left[\begin{array}{lll}\boldsymbol{v}_{\mathcal{L}}^{i} \otimes & \boldsymbol{v}_{1}^{i} \\ \boldsymbol{v}_{\mathcal{L}}^{i} \otimes & \boldsymbol{v}_{2}^{i}\end{array}\right]$.

It is well known in [19] that there is only one zero eigenvalue in $\mathcal{L}_{n}, \lambda_{\mathcal{L}}^{i}=0$, when the graph $\mathcal{G}$ satisfies Assumption 1. In this case, $\psi_{i}$ has eigenvalues $\lambda_{A}$ and $\lambda_{\bar{A}}$ associated with eigenvectors $\left[\begin{array}{c}\mathbf{0} \\ \boldsymbol{v}_{A}\end{array}\right]$ and $\left[\begin{array}{c}\boldsymbol{v}_{\bar{A}} \\ \left(\lambda_{\bar{A}} I_{n}-A\right)^{-1} B \bar{C} \boldsymbol{v}_{\bar{A}}\end{array}\right]$ respectively since $\psi_{i}=\left[\begin{array}{cc}\bar{A} & 0 \\ B \bar{C} & A\end{array}\right]$, where $\lambda_{A}$ and $\lambda_{\bar{A}}$ are the eigenvalues of $A$ and $\bar{A}, \boldsymbol{v}_{A}$ and $\boldsymbol{v}_{\bar{A}}$ are the corresponding eigenvectors of $A$ and $\bar{A}$, respectively. This also shows that eigenvalues of $\Psi$ include $\lambda_{A}$ and $\lambda_{\bar{A}}$ with the associated eigenvectors being $\left[\begin{array}{c}\mathbf{0} \\ \mathbf{1} \otimes \boldsymbol{v}_{A}\end{array}\right]$ and $\left[\begin{array}{c}\mathbf{1} \otimes \boldsymbol{v}_{\bar{A}} \\ \mathbf{1} \otimes\left(\lambda_{\bar{A}} I_{n}-A\right)^{-1} B \bar{C} \boldsymbol{v}_{\bar{A}}\end{array}\right]$. It is worth noting that the invertibility of $A-\lambda_{\bar{A}} I_{n}$ follows since an SNI controller can always be chosen such that $\lambda_{\bar{A}} \neq \lambda_{A}$.

In the case of $\lambda_{\mathcal{L}}^{i}>0$ and $\operatorname{det}(A) \neq 0$, it can be shown in a similar manner as Theorem 5 of [4] that

$$
\begin{aligned}
\psi_{i} & =\left[\begin{array}{cc}
\bar{A}+\lambda_{\mathcal{L}}^{i} \bar{B} D \bar{C} & \lambda_{\mathcal{L}}^{i} \bar{B} C \\
B \bar{C} & A+\lambda_{\mathcal{L}}^{i} B \bar{D} C
\end{array}\right] \\
& =\left[\begin{array}{cc}
\bar{A} & 0 \\
B \bar{C} & A
\end{array}\right]+\lambda_{\mathcal{L}}^{i}\left[\begin{array}{c}
\bar{B} \\
B \bar{D}
\end{array}\right]\left[\begin{array}{ll}
D \bar{C} & C
\end{array}\right]=\Phi T
\end{aligned}
$$

where $T=\left[\begin{array}{cc}\bar{Y}^{-1}-\lambda_{\mathcal{L}}^{i} \bar{C}^{*} D \bar{C} & -\lambda_{\mathcal{L}}^{i} \bar{C}^{*} C \\ -C^{*} \bar{C} & Y^{-1}-\lambda_{\mathcal{L}}^{i} C^{*} \bar{D} C\end{array}\right]$ and $\Phi=$ $\left[\begin{array}{cc}\bar{A} \bar{Y} & 0 \\ 0 & A Y\end{array}\right] \cdot \psi_{i}$ is Hurwitz if and only if $\bar{\lambda}\left(P(0) P_{s}(0)\right)<\frac{1}{\lambda_{\mathcal{L}}^{i}}$, which coincides with the condition in Theorem 1 when $\lambda_{\mathcal{L}}^{i}=\bar{\lambda}\left(\mathcal{L}_{n}\right)$.

In the case of $\lambda_{\mathcal{L}}^{i}>0$ and $\operatorname{det}(A)=0$, it can be verified in a similar manner as [15] that

$$
\psi_{i}=\left[\begin{array}{cc}
\bar{A} & \lambda_{\mathcal{L}}^{i} \bar{B} C \\
B \bar{C} & A+\lambda_{\mathcal{L}}^{i} B \bar{D} C
\end{array}\right]
$$

due to $D=0 . \psi_{i}$ is also Hurwitz when the conditions 2) and 3) in Lemma 4 hold. A detailed proof is omitted due to page limitations.

One direct observation from the above analysis: the number of eigenvalues of $\Psi$ on the imaginary axis is equal to the number of eigenvalues of $A$ on the imaginary axis and all of the other eigenvalues lie in the OLHP since $\bar{A}$ is Hurtwiz [14]. Thus, the steady state of the closed-loop system (11) in general depends only on the eigenvalues of $A$ on the imaginary axis as shown in the following theorem:

Theorem 2: Given the closed-loop system in (11), the steady state can be expressed in the general form

$$
\left[\begin{array}{c}
\overline{\boldsymbol{x}}(t) \\
\boldsymbol{x}(t)
\end{array}\right] \stackrel{t \rightarrow \infty}{\longrightarrow}\left[\begin{array}{lll}
\boldsymbol{w}_{j}, & \cdots, & \boldsymbol{w}_{k}^{g}
\end{array}\right] e^{J^{\prime} t}\left[\begin{array}{c}
\boldsymbol{v}_{j}^{T} \\
\vdots \\
\boldsymbol{v}_{k}^{g T}
\end{array}\right]\left[\begin{array}{c}
\overline{\boldsymbol{x}}(0) \\
\boldsymbol{x}(0)
\end{array}\right],
$$

where $J^{\prime}$ is the Jordan block associated with $n_{0}$ eigenvalues of $\Psi$ on the imaginary axis denoted by $\lambda_{A}, \boldsymbol{w}_{j}$ and $\boldsymbol{v}_{j}$ are the right and left eigenvector of $\Psi$ associated with $\lambda_{A}$ given by

$$
\boldsymbol{w}_{j}=\left[\begin{array}{c}
\mathbf{0} \\
\mathbf{1} \otimes \boldsymbol{v}_{A}^{r}
\end{array}\right], \boldsymbol{v}_{j}=\left[\begin{array}{c}
\mathbf{1} \otimes\left(\frac{1}{n}\left(\lambda_{A} I_{q}-\bar{A}\right)^{-1} \bar{C}^{T} B^{T} \boldsymbol{v}_{A}^{l}\right) \\
\mathbf{1} \otimes \frac{1}{n} \boldsymbol{v}_{A}^{l}
\end{array}\right],
$$

$\forall j=1, \cdots, n_{0}-\left(n_{a}-n_{g}\right)$, where $n_{a}$ and $n_{g}$ denote the algebraic and geometric multiplicity of $\lambda_{A}$ respectively. $\boldsymbol{v}_{A}^{r}, \boldsymbol{v}_{A}^{l}$ are the right and left eigenvectors of $A$ associated with $\lambda_{A}$. Moreover, in the case that $n_{a}>n_{g}, \boldsymbol{w}_{k}^{g}$ and $\boldsymbol{v}_{k}^{g}$ are the generalised right and left eigenvectors given by

$$
\boldsymbol{w}_{k}^{g}=\left[\begin{array}{c}
\mathbf{0} \\
\mathbf{1} \otimes \boldsymbol{v}_{A}^{r_{g}}
\end{array}\right], \boldsymbol{v}_{k}^{g}=\left[\begin{array}{c}
\mathbf{1} \otimes\left(\frac{1}{n}\left(\lambda_{A} I_{q}-\bar{A}\right)^{-1} \bar{C}^{T} B^{T} \boldsymbol{v}_{A}^{l_{g}}\right) \\
\mathbf{1} \otimes \frac{1}{n} \boldsymbol{v}_{A}^{l_{g}}
\end{array}\right],
$$

where $k=1, \cdots, n_{a}-n_{g}, \boldsymbol{v}_{A}^{r_{g}}$ and $\boldsymbol{v}_{A}^{l_{g}}$ are the generalised right and left eigenvectors of $A$ associated with $\lambda_{A}$.

Proof: It is straightforward that $\left[\begin{array}{l}\overline{\boldsymbol{x}}(t) \\ \boldsymbol{x}(t)\end{array}\right]=e^{\Psi t}\left[\begin{array}{l}\overline{\boldsymbol{x}}(0) \\ \boldsymbol{x}(0)\end{array}\right]=$ $P e^{J t} P^{-1}\left[\begin{array}{l}\overline{\boldsymbol{x}}(0) \\ \boldsymbol{x}(0)\end{array}\right] t \rightarrow \infty P\left[\begin{array}{cc}e^{J^{\prime} t} & 0 \\ 0 & 0\end{array}\right] P^{-1}\left[\begin{array}{l}\overline{\boldsymbol{x}}(0) \\ \boldsymbol{x}(0)\end{array}\right]$, where $J_{r \times r}^{\prime}$ is the Jordan block associated with $n_{0}$ eigenvalues on the imaginary axis. Also, $P=\left[\boldsymbol{w}_{1}, \cdots, \boldsymbol{w}_{n_{0}}, \cdots, \boldsymbol{w}_{(p+q) n}\right]$, where $\boldsymbol{w}_{i}$ is the right eigenvector of $\Psi$ and $P^{-1}=\left[\boldsymbol{v}_{1}, \cdots, \boldsymbol{v}_{n_{0}}, \cdots, \boldsymbol{v}_{(p+q) n}\right]^{T}$, where $\boldsymbol{v}_{i}$ is the left eigenvector of $\Psi$.

It can be found, without loss of generality, that the right and left eigenvectors of $\Psi$ associated with the eigenvalues on imaginary axis are given in (15). Thereby, the steady state generally converges to

$$
\left[\begin{array}{c}
\overline{\boldsymbol{x}}(t) \\
\boldsymbol{x}(t)
\end{array}\right] \stackrel{t \rightarrow \infty}{\longrightarrow}\left[\begin{array}{lll}
\boldsymbol{w}_{1}, & \cdots, & \boldsymbol{w}_{n_{0}}
\end{array}\right] e^{J^{\prime} t}\left[\begin{array}{c}
\boldsymbol{v}_{1}^{T} \\
\vdots \\
\boldsymbol{v}_{n_{0}}^{T}
\end{array}\right]\left[\begin{array}{c}
\overline{\boldsymbol{x}}(0) \\
\boldsymbol{x}(0)
\end{array}\right]
$$

However, in the case that $n_{a}>n_{g}$, the generalised right and left eigenvectors are given in (16). Thus, the steady state converges to (14) instead of (17).

Next, convergence sets of several special cases of NI systems are given in detail:

Corollary 1: In the case that the NI plant is a single-integrator, i.e., $\dot{x}_{i}=u_{i}, y_{i}=x_{i}$, the convergence set of (11) is $y_{s s}=-\bar{C} \bar{A}^{-T}$. ave $(\overline{\boldsymbol{x}}(0))+$ ave $(\boldsymbol{x}(0))$.

Proof: The convergence set can be obtained by noting the eigenvectors $\boldsymbol{w}_{j}=\left[\begin{array}{ll}\mathbf{0}^{T} & \mathbf{1}_{n}^{T}\end{array}\right]^{T}, \boldsymbol{v}_{j}=\left[\begin{array}{ll}-\frac{1}{n} \bar{C} \bar{A}^{-T} \mathbf{1}_{n}^{T} & \frac{1}{n} \mathbf{1}_{n}^{T}\end{array}\right]^{T}$ and applying (17) in Theorem 2. 
Corollary 2: In the case that the NI plant is a double-integra i.e., $\dot{\xi}_{i}=\zeta_{i}, \dot{\zeta}_{i}=u_{i}, y_{i}=\xi_{i}$, the convergence set of $(11$ $y_{s s}=-\bar{C} \bar{A}^{-T} \cdot \operatorname{ave}(\overline{\boldsymbol{x}}(0)) t+\operatorname{ave}(\boldsymbol{\xi}(0))+\operatorname{ave}(\boldsymbol{\zeta}(0)) t$.

Proof: For double-integrator plants, $n_{a}=2>1=$ for $\lambda(A)=0$. The convergence set is straightforward by $\mathrm{r}$ ing $\boldsymbol{w}_{j}=\left[\begin{array}{lll}\mathbf{0}^{T} & \mathbf{1}_{n}^{T} & \mathbf{0}^{T}\end{array}\right]^{T}, \boldsymbol{v}_{j}=\left[\begin{array}{lll}\mathbf{0}^{T} & \frac{1}{n} \mathbf{1}_{n}^{T} & \mathbf{0}^{T}\end{array}\right]^{T}, \boldsymbol{w}_{k}^{g}$ $\left[\begin{array}{lll}\mathbf{0}^{T} & \mathbf{0}^{T} & \mathbf{1}_{n}^{T}\end{array}\right]^{T}, \boldsymbol{v}_{k}^{g}=\left[\begin{array}{lll}-\frac{1}{n} \bar{C} \bar{A}^{-T} \mathbf{1}_{n}^{T} & \mathbf{0}^{T} & \frac{1}{n} \mathbf{1}_{n}^{T}\end{array}\right]^{T}$ after $\mathrm{r} \mathrm{s}$ ranging $\boldsymbol{x}=\left[\begin{array}{ll}\boldsymbol{\xi}^{T} & \boldsymbol{\zeta}^{T}\end{array}\right]^{T}$ and applying (14).

Corollary 3: In the case that the NI plant is a damped flexi structure, the convergence set of (11) is $y_{s s}=0$.

Proof: This is straightforward and thus omitted.

\section{ILlustrative EXAmples}

In this section, numerical examples of typical NI systems are given to illustrate the main results of this technical note. A scenario of 3 NI systems is considered and the communication graph $\mathcal{G}$ is given as in Fig. 3. Therefore, the Laplacian matrix of $\mathcal{G}$ can be derived according to the definition in Section I:

$$
\begin{aligned}
& \mathcal{G} \longrightarrow 3 \\
& \mathcal{G}
\end{aligned} \mathcal{L}_{3}=\left[\begin{array}{ccc}
1 & -1 & 0 \\
-1 & 2 & -1 \\
0 & -1 & 1
\end{array}\right]
$$

Fig. 3. Communication topology $\mathcal{G}$ and associated Laplacian matrix

\section{A. Multiple Single-Integrator Systems}

Suppose that the NI systems have identical single-integrator namics as shown in Corollary 1 with the initial condition be $\boldsymbol{x}(0)=\left[\begin{array}{lll}1 & 2 & 3\end{array}\right]^{T}$. The SNI controller is designed as indica in Theorem 1 to be $\bar{A}=-2, \bar{B}=1, \bar{C}=1, \bar{D}=$ with the initial condition being $\overline{\boldsymbol{x}}(0)=\left[\begin{array}{lll}0.1 & 0.2 & 0.3\end{array}\right]^{T}$. Without considering disturbances firstly, it can be verified as Corollary 1 that $y_{s s}=-\bar{C} \bar{A}^{-T}$ ave $(\overline{\boldsymbol{x}}(0))+\operatorname{ave}(\boldsymbol{x}(0))=\frac{1}{2} * 0.2+2=2.1$, which is shown at the top left of Fig. 4. If external disturbances are inserted, robust output feedback consensus is also achieved with the steady state consensus value perturbed by filtered disturbances as shown at the top right of Fig. 4. The robust performance of the control law can be improved by tuning the SNI controller to, for example $\bar{D}=-5$, which are shown in the bottom left and right of Fig. 4, respectively.

One may notice that when the initial condition of the controller $\overline{\boldsymbol{x}}(0)$ is set to $\mathbf{0}$ (a reasonable choice as the controller is determined by the designer), the convergence set naturally becomes the centroid of the initial pattern, i.e., $y_{s s}=\operatorname{ave}(\boldsymbol{x}(0))$, which in turn implies that the result for the average consensus protocol in [19] is a special case of the proposed result. Alternatively, the desired convergence point can be chosen by properly initialising the SNI controller, which can be seen as a more general result.

\section{B. Multiple Double-Integrator Systems}

Suppose that the NI systems have identical double-integrator dynamics as shown in Corollary 2 with the initial conditions being $\boldsymbol{\xi}(0)=\left[\begin{array}{lll}1 & 2 & 3\end{array}\right]^{T}, \boldsymbol{\zeta}(0)=\left[\begin{array}{lll}0.1 & 0.2 & 0.3\end{array}\right]^{T}$. The same SNI controller can be adopted as in Subsection IV.A. Without considering disturbances firstly, it can be verified using Corollary 2 that $y_{s s}=\xi_{i}(\infty)=$ $-\bar{C} \bar{A}^{-T} \cdot \operatorname{ave}(\overline{\boldsymbol{x}}(0))+\operatorname{ave}(\boldsymbol{\xi}(0))+\operatorname{ave}(\boldsymbol{\zeta}(0)) t=\frac{1}{2} * 0.2+2+0.2 t=$ $2.1+0.2 t$ and $\zeta_{i}(\infty)=-\bar{C} \bar{A}^{-T} \cdot \operatorname{ave}(\overline{\boldsymbol{x}}(0))+\operatorname{ave}(\boldsymbol{\zeta}(0))=$ $\frac{1}{2} * 0.2+0.2=0.3$, which is exactly as shown at the top of Fig. 5 . If the same disturbances as in Subsection IV.A are inserted, output consensus is also achieved with the steady state values perturbed
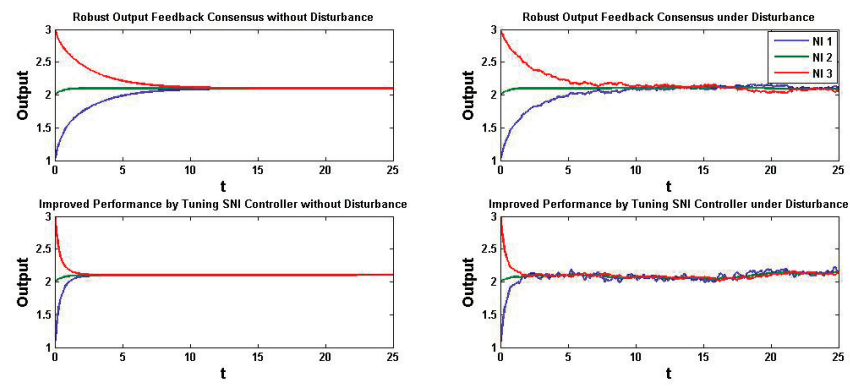

Fig. 4. Robust output consensus for networked single-integrator systems

by filtered disturbances as shown at the bottom of Fig. 5. Again, appropriate choices of the SNI controller can be made to minimise the effects of external disturbances, which is omitted here due to the page limitations.

One can also choose the initial condition of the controller to be $\overline{\boldsymbol{x}}(0)=\mathbf{0}$ to obtain the natural convergence set as $y_{s s}=\xi_{s s}=$ $\operatorname{ave}(\boldsymbol{\xi}(0))+\operatorname{ave}(\boldsymbol{\zeta}(0)) t$ and $\zeta_{s s}=\operatorname{ave}(\boldsymbol{\zeta}(0))$. The same conclusion can hence be drawn as in Subsection IV.A.
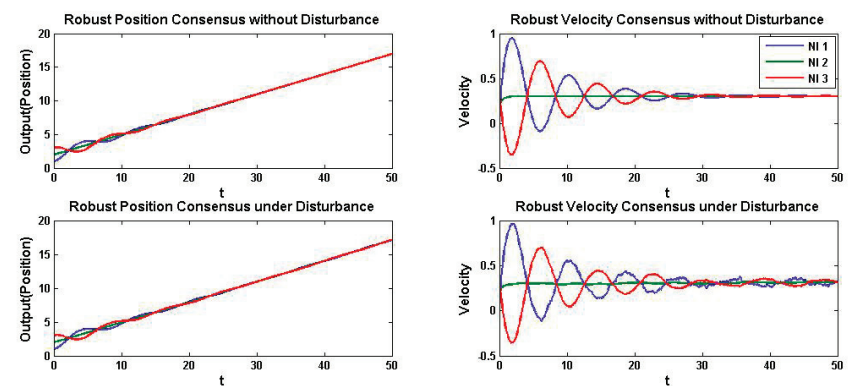

Fig. 5. Robust output consensus for networked double-integrator systems

\section{Multiple Flexible Structures Systems}

Suppose that the NI systems are damped flexible structures as shown for example in Fig. 2 of [4]: $M \ddot{\boldsymbol{x}}_{i}+C \dot{\boldsymbol{x}}_{i}+K \boldsymbol{x}_{i}=$ $\boldsymbol{u}_{i}, \boldsymbol{y}_{i}=\boldsymbol{x}_{i}, \quad i=1, \cdots, 3$ where $\boldsymbol{x}_{i}=\left[\begin{array}{ll}x_{i}^{1 T} & x_{i}^{2 T}\end{array}\right]^{T}, \quad \boldsymbol{u}_{i}=$ $\left[\begin{array}{ll}u_{i}^{1 T} & u_{i}^{2^{T}}\end{array}\right]^{T}, M=\operatorname{diag}\left(m_{1}, m_{2}\right), C=\left[\begin{array}{cc}c_{1}+c & -c \\ -c & c_{2}+c\end{array}\right], K=$ $\left[\begin{array}{cc}k_{1}+k & -k \\ -k & k_{2}+k\end{array}\right]$ with $m_{1}=1, m_{2}=0.5, k_{1}=k_{2}=k=1$ and $c_{1}=c_{2}=c=0.1$. The initial conditions are given as $\boldsymbol{x}(0)=\left[\begin{array}{llllll}1 & 2 & 3 & 4 & 5 & 6\end{array}\right]^{T}$ and $\dot{\boldsymbol{x}}(0)=\left[\begin{array}{llllll}0.1 & 0.2 & 0.3 & 0.4 & 0.5 & 0.6\end{array}\right]^{T}$. The SNI controller can be designed as indicated in Theorem 1 to be $\bar{A}=-4 I_{2}, \bar{B}=I_{2}, \bar{C}=I_{2}, \bar{D}=0_{2}$ since $\bar{\lambda}(P(0))=1$ and thus $\bar{\lambda}\left(P(0) P_{s}(0)\right)=\frac{1}{4}<\frac{1}{\bar{\lambda}\left(\mathcal{L}_{n}\right)}=\frac{1}{3}$ with the initial condition being $\left[\begin{array}{llllll}0 & 0 & 0 & 0 & 0 & 0\end{array}\right]^{T}$. Robust output feedback consensus can be achieved as shown at the top of Fig. 6 under external disturbances, which also validates Corollary 3.

If the NI systems are considered as undamped flexible structures as shown in Fig. 2 of [14], which correspond to the above damped flexible structure dynamics without the damping term $C$, robust output feedback consensus can be achieved as shown at the bottom of Fig. 6 under external disturbances.

\section{CONCLUSION}

NI systems include a wide range of LTI systems that are commonly studied in the consensus literature. This class of systems 

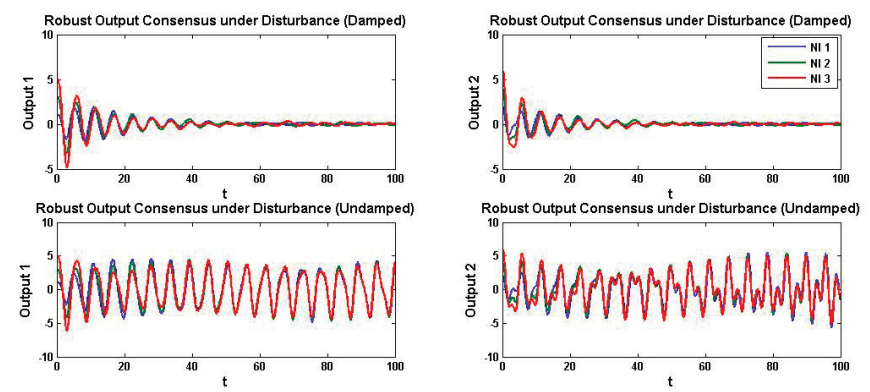

Fig. 6. Robust output consensus for networked flexible structures

and corresponding theory also include a large class of dynamical systems that have not been studied in consensus literature to date. The robust output feedback consensus problem for this class of systems is hence of interest. The advantage of using NI systems theory for solving the consensus problem is four-fold: (a) it only uses output feedback information as opposed to full-state feedback information; (b) it provides robustness guarantees w.r.t. $\mathfrak{L}_{2}$ external disturbance; (c) it allows tuning of a whole class of SNI control laws for performance; and (d) it bypasses traditional searches for Lyapunov candidate functions. In addition, the characterised convergence set also makes it possible to initialise the controller state to achieve the desired final consensus target.

Future research directions include robust output feedback consensus for networked heterogeneous NI systems as well as the impact of switching topologies and time delays.

\section{ACKNOWLEDGMENT}

The financial support of the Engineering and Physical Sciences Research Council, the UK Department for Business, Innovation and Skills, the Australian Research Council, the Excellent Young Scholars Research Fund of Beijing Institute of Technology as well as the Beijing Institute of Technology Research Fund Program for Young Scholars is gratefully acknowledged.

\section{REFERENCES}

11] Z. Li, Z. Duan, G. Chen, and L. Huang, "Consensus of multiagent systems and synchronization of complex networks: A unified view point," IEEE Trans. on Circuits and Systems I: Regular Papers, vol. 57, no. 1, pp. 213-224, 2010.

[2] Z. Song, A. Lanzon, S. Patra, and I. Petersen, "A negative-imaginary lemma without minimality assumptions and robust state-feedback synthesis for uncertain negative-imaginary systems," Systems and Control Letters, vol. 61, no. 12, pp. 1269-1276, 2012.

[3] J. Xiong, I. Petersen, and A. Lanzon, "On lossless negative imaginary systems," Automatica, vol. 48, no. 6, pp. 1213-1217, 2012.

[4] A. Lanzon and I. Petersen, "Stability robustness of a feedback interconnection of systems with negative imaginary frequency response," IEEE Trans. Autom. Control, vol. 53, no. 4, pp. 1042-1046, 2008.

[5] M. A. Mabrok, A. G. Kallapur, I. Petersen, and A. Lanzon, "Spectral conditions for negative imaginary systems with applications to nanopositioning," IEEE/ASME Transactions on Mechatronics, vol. 19, no. 3, pp. 895-903, 2014.

[6] I. Petersen and A. Lanzon, "Feedback control of negative-imaginary systems," IEEE Control Systems Magazine, vol. 30, no. 5, pp. 54-72, 2010.

[7] S. Patra and A. Lanzon, "Stability analysis of interconnected systems with 'mixed' negative-imaginary and small-gain properties," IEEE Trans. Autom. Control, vol. 56, no. 6, pp. 1395-1400, 2011.

[8] N. Chopra and W. Spong, "Output synchronization of nonlinear systems with relative degree one," Recent Adv. Learning Control, vol. 371, pp. 51-64, 2008.

[9] Y. Su, Y. Hong, and J. Huang, "A general result on the robust cooperative output regulation for linear uncertain multi-agent systems," IEEE Trans. Autom. Control, vol. 58, no. 5, pp. 1275-1279, 2013.
[10] C. Ma and J. Zhang "Necessary and sufficient conditions for consensusability of linear multi-agent systems," IEEE Trans. on Autom. Control, vol. 55, no. 5, pp. 1263-1268, 2010.

[11] J. Seo, H. Shim, and J. Back, "Consensus of high-order linear systems using dynamic output feedback compensator: Low gain approach," Automatica, vol. 45, no. 11, pp. 2659-2664, 2009.

[12] L. Zhu and Z. Chen, "Robust homogenization and consensus of nonlinear multi-agent systems," Systems and Control Letters, vol. 65, pp. 50-55, 2014.

[13] H. Kim, H. Shim, and J. Seo, "Output consensus of heterogeneous uncertain linear multi-agent systems," IEEE Trans. Autom. Control, vol. 56, no. 1, pp. 200-206, 2011.

[14] J. Xiong, I. Petersen, and A. Lanzon, "A negative imaginary lemma and the stability of interconnections of linear negative imaginary systems," IEEE Trans. Autom. Control, vol. 55, no. 10, pp. 2342-2347, 2010.

[15] M. Mabrok, A. Kallapur, I. Petersen, and A. Lanzon, "Generalizing negative imaginary systems theory to include free body dynamics: Control of highly resonant structures with free body motion," arXiv: 1305.1079. IEEE Trans. Autom. Control, vol. 59, no. 10, pp. 2692-2707, 2014.

[16] A. Laub, Matrix Analysis for Scientists and Engineers. Philadelphia, PA: Society for Industrial and Applied Mathematics, 2005

[17] K. Zhou, J. Doyle, and K. Glover, Robust and Optimal Control. Englewood Cliffs: NJ: Prentice-Hall, 1996.

[18] R. Grone and R. Merris, "The laplacian spectrum of a graph ii," SIAM Journal on Discrete Mathematics, vol. 7, no. 2, pp. 221-229, 1994.

[19] W. Ren and R. W. Beard, Distributed Consensus in Multi-vehicle Cooperative Control. London: Communications and Control Engineering Serie, Springer-Verlag, 2008. 